\title{
NOVEL AND NON-STANDARD ACCELERATION TECHNOLOGIES EXCITATION OF THE WAKE WAVES BY A LASER PULSE IN ION DIELECTRICS
}

\author{
V.A. Balakirev, I.N. Onishchenko \\ National Science Center "Kharkov Institute of Physics and Technology", Kharkiv, Ukraine \\ E-mail: onish@kipt.kharkov.ua
}

The process of excitation of Cherenkov electromagnetic radiation by a laser pulse in ion dielectric waveguide is investigated. Nonlinear electric polarization in isotropic ion dielectric medium and, accordingly, polarization charges and currents induced by a ponderomotive force of a laser pulse are determined. Frequency spectra of the excited wakefields in the infrared and microwave frequency ranges are obtained. The spatio-temporal structure of the wakefield in ion dielectric waveguide is obtained and studied. It is shown that the excited field consists of a potential polarization electric field, as well as a set of eigen electromagnetic waves of ion dielectric waveguide.

PACS: 41.75.Lx, 41.85.Ja, 41.69.Bq

\section{INTRODUCTION}

A effect of Cherenkov radiation takes place when a high-power laser pulse propagates in a dielectric [1 - 5]. A necessary condition for the appearance of a Cherenkov radiation of a laser pulse is that the group velocity of the laser pulse must exceed the phase velocity of the radiated electromagnetic wave. The effect of Cherenkov radiation of a laser pulse in a dielectric medium is as follows. When a laser pulse propagates in a dielectric a pulsed ponderomotive force quadratic in the laser field propagating in the medium with the group velocity of the laser pulse will act on the bonded electrons of the atoms (ions) of a medium. This force, in turn, will lead to the polarization of the atoms (ions) of the dielectric. Induced polarization charges and currents will coherently radiate electromagnetic waves (Cherenkov radiation). The effect of the Cherenkov radiation of a laser pulse is quite similar to the Cherenkov radiation of an electron bunch moving in a dielectric medium, with the difference that the ponderomotive force of the laser pulse plays the role of the pulse electric field of the electron bunch.

The Cherenkov wakefield radiation in a dielectric medium of a high-power ultrashort laser pulse can be used to accelerate charged particles similarly to a laserplasma wakefield acceleration method [6].

A wider class of dielectrics is formed by ion-bonded dielectrics [7 - 9]. No pure element of the periodic table is related to dielectrics of this class. All ion dielectrics are chemical compounds. Ion crystals are composed of positive and negative ions. These ions form a crystal lattice as a result of Coulomb attraction of oppositely charged ions.

The traditional example of ion dielectrics are crystals of an alkali-halide group with the formula $A_{I} B_{V I I}$ (for example, $\mathrm{NaCl}$ and $\mathrm{KCl}$ ). In crystals of this group, it is energetically advantageous for an atom of alkali metal to transfer its valence electron to an adjacent halide atom and fill its outer shell. As a result, an ion bond arises between the atoms of different elements. This bond is due to the interaction of oppositely charged ions. Below we restrict consideration to the simplest case of diatomic crystals.
In determining the total electric polarization induced by a laser pulse in an ion dielectric, it is necessary to take into account both the total contribution of the polarizations of the electron shells of all the ions which form the crystal and the total contribution of the positive and negative ions of the crystal.

In this paper, a system of nonlinear equations of macroscopic electrodynamics is formulated, which describes the process of excitation of Cherenkov radiation by a laser pulse in an ion dielectric medium.

On the basis of these equations, the effect of the Cherenkov radiation of a laser pulse in a dielectric waveguide (light guide) will be investigated. A complete picture of the excitation of Cherenkov radiation by a laser pulse propagating in an ion dielectric is presented. The frequency spectrum of Cherenkow radiation is determined. The spatio-temporal structure of the Cherenkov electromagnetic field has been obtained and studied. Note that when the laser pulse crosses the dielectric boundary, the transition radiation effect is possible by analogy with the case of an electron bunch [10].

\section{PROBLEM STATEMENT. BASIC EQUATIONS}

A laser pulse (wave packet) with electromagnetic field components propagates in a homogeneous dielectric medium

$$
\begin{aligned}
\vec{E}_{L}(\vec{r}, t) & =\frac{1}{2} \vec{E}_{0}(\vec{r}, t) e^{i \psi_{L}}+c . c . \\
\vec{H}_{L}(\vec{r}, t) & =\frac{1}{2 i k_{0}} \operatorname{rot}\left[\vec{E}_{0}(\vec{r}, t) e^{i \psi_{L}}\right]+\text { c.c. }
\end{aligned}
$$

$\psi_{L}=\vec{k}_{L} \vec{r}-\omega_{L} t, \vec{k}_{L}$ is wave vector; $k_{0}=\omega_{L} / c, \omega_{L}$ is carrier frequency of a laser pulse; $\vec{E}_{0}(\vec{r}, t)$ is a laser pulse envelope slowly varying in space and time.

Under the action of the ponderomotive force (RFpressure force) a polarization arises in the dielectric, slow on the carrier frequency scale, which in turn is the source of the electromagnetic field of the laser pulse (Cherenkov radiation). Maxwell's system of equations describing the electromagnetic field, which is excited by a polarization induced by a laser pulse, has the form

$$
\operatorname{rot} \vec{E}=-\frac{1}{c} \frac{\partial \vec{H}}{\partial t}, \quad \operatorname{rot} \vec{H}=\frac{1}{c} \frac{\partial \vec{E}}{\partial t}+\frac{4 \pi}{c} \frac{\partial \vec{P}}{\partial t},
$$




$$
\operatorname{div} \vec{E}=-4 \pi \operatorname{div} \vec{P}, \quad \operatorname{div} \vec{H}=0,
$$

$\vec{P}$ is vector of electric polarization.

In ion dielectrics there are two mechanisms of electric polarization. This is primarily an electron polarization mechanism inherent in all types of dielectrics. Electron polarization is due to the displacement of a shell of bound electrons relative to their nuclei under the action of an electric field. The second polarization mechanism is ionic; it is caused by the relative displacement of oppositely charged ions.

First of all, we formulate equations describing the electron polarization of diatomic ion crystals induced by a laser pulse.

In a condensed medium, each atom is in a local (acting) electric field $\overrightarrow{\mathrm{E}}_{\text {loc }}$, which can differ substantially from the macroscopic field $\overrightarrow{\mathrm{E}}$ included in Maxwell's equations (2). The local electric field $\overrightarrow{\mathrm{E}}_{\text {loc }}$ includes both the external electric field and the total electric field of the induced dipoles surrounding a given atom (ion). In a crystal medium with a cubic crystal lattice, the local electric field is described by the Lorentz formula [7 - 9]

$$
\overrightarrow{\mathrm{E}}_{\mathrm{loc}}=\overrightarrow{\mathrm{E}}+\frac{4 \pi}{3} \overrightarrow{\mathrm{P}} \text {. }
$$

Taking into account the local field effect, the expression for the ponderomotive force acting on the electrons of the crystal ion shell from the side of the laser pulse has the form [3 - 5]

$$
\begin{gathered}
\overrightarrow{\mathrm{F}}_{\text {pon }}^{( \pm)}=\frac{\mathrm{e}^{2}}{4 \mathrm{~m}} \frac{\varepsilon_{\mathrm{L}}+2}{3} \frac{1}{\omega_{\mathrm{de}( \pm)}^{2}-\omega_{\mathrm{L}}^{2}} \vec{\Pi} \\
\vec{\Pi}=\nabla\left|\overrightarrow{\mathrm{E}}_{0}\right|^{2}+\frac{\varepsilon_{\mathrm{L}}-1}{3}\left[\left(\overrightarrow{\mathrm{E}}_{0}^{*} \nabla\right) \overrightarrow{\mathrm{E}}_{0}+\left(\overrightarrow{\mathrm{E}}_{0} \nabla\right) \overrightarrow{\mathrm{E}}_{0}^{*}\right] .
\end{gathered}
$$

The indexes $( \pm)$ correspond to positive and negative ions, $\omega_{d e( \pm)}$ are frequencies of the dipole oscillations of the electron shells of ions, $\varepsilon_{L}$ is the dielectric constant of the medium at the frequency of the laser pulse. The first term in (5) describes the gradient force of HFpressure. The second term appears only in the case of a crystal medium and is caused by the difference between the local electric field in a crystal and the average electric field of a laser pulse in dielectric medium. In dielectric media where the active field coincides with the external field, for example, in the gas dielectric or plasma this term is absent.

Under the action of ponderomotive force in dielectric electron and ion polarizations appear. Full polarization of ion dielectrics is

$$
\vec{P}=\vec{P}_{e}^{(+)}+\vec{P}_{e}^{(-)}+\vec{P}_{i},
$$

where $\vec{P}_{e}^{( \pm)}$are the partial electron polarizations of positive and negative ions; $\vec{P}_{i}$ is ion polarization. Partial polarizations are described by a system of coupled linear oscillators

$$
\begin{array}{r}
\frac{\partial^{2} \vec{P}_{e}^{(+)}}{\partial t^{2}}+\omega_{d e+}^{2} \vec{P}_{e}^{(+)}-\frac{1}{3} \omega_{p e+}^{2}\left(\vec{P}_{e}^{(+)}+\vec{P}_{e}^{(-)}+\vec{P}_{i}\right)= \\
=\frac{1}{4 \pi} \omega_{p e+}^{2} \vec{E}-\frac{e N_{0}}{4 m} \frac{\varepsilon_{L}+2}{3} \alpha_{L}^{(+)} \vec{\Pi}
\end{array}
$$

$$
\begin{array}{r}
\frac{\partial^{2} \vec{P}_{e}^{(-)}}{\partial t^{2}}+\omega_{d e-}^{2} \vec{P}_{e}^{(-)}-\frac{1}{3} \omega_{p e-}^{2}\left(\vec{P}_{e}^{(+)}+\vec{P}_{e}^{(-)}+\vec{P}_{i}\right)= \\
=\frac{1}{4 \pi} \omega_{p e-}^{2} \vec{E}-\frac{e N_{0}}{4 m} \frac{\varepsilon_{L}+2}{3} \alpha_{L}^{(-)} \vec{\Pi}, \\
\frac{\partial^{2} \vec{P}_{i}}{\partial t^{2}}+\omega_{d i}^{2} \vec{P}_{i}-\frac{1}{3} \omega_{p i}^{2}\left(\vec{P}_{e}^{(+)}+\vec{P}_{e}^{(-)}+\vec{P}_{i}\right)=\frac{1}{4 \pi} \omega_{p i}^{2} \vec{E},
\end{array}
$$

where $\alpha_{L}^{( \pm)}$are electron polarizabilities of individual positive and negative ions at laser pulse frequencies; $\omega_{p e( \pm)}^{2}=4 \pi e q^{( \pm)} N_{0} / m$ is square of the effective electron plasma frequency; $q^{( \pm)}$is full charge of the electron shell of the corresponding ion; $N_{0}$ is concentration of ions of each type; $\omega_{d i}$ is the eigen frequency of ion dipole oscillations; $\omega_{p i}^{2}=4 \pi q_{i}^{2} N_{0} / M$ is the square of the ion plasma frequency; $M$ is reduced mass of ions; $q_{i}$ is ion charge.

The left-hand sides of equations (7) for electron polarizations include complete polarization of the ion dielectric, which also includes ion polarization $\vec{P}_{i}$. Note that since the ponderomotive force acting on ions is inversely proportional to the mass of the ions, then it is small and we neglected it in equation (7).

Thus, partial polarizations are described by a system of coupled linear oscillators. The external force exciting these oscillators is the ponderomotive force from the side of the laser pulse.

The Maxwell equations (2), together with the equations for partial polarizations (7) and the relation (6) for the full polarization, are closed and describe the Cherenkov excitation of electromagnetic radiation of a laser pulse in an ion dielectric.

We will solve this system of equations by the method of Fourier transform

$$
\vec{E}(\vec{r}, t)=\int_{-\infty}^{\infty} \vec{E}_{\omega}(r) e^{-i \omega t} d \omega, \vec{P}(\vec{r}, t)=\int_{-\infty}^{\infty} \vec{P}_{\omega}(r) e^{-i \omega t} d \omega,
$$

where $\vec{E}_{\omega}(r), \vec{P}_{\omega}(\vec{r})$ are Fourier-components of the corresponding quantities. From the system of coupled equations for partial polarizations (7) we find the expression for the Fourier components of the full polarization vector

$$
\vec{P}_{\omega}=\frac{\varepsilon(\omega)-1}{4 \pi} \vec{E}_{\omega}-\mu \vec{\Pi}_{\omega},
$$

where

$$
\varepsilon(\omega)=\frac{1+\frac{2}{3} \Lambda(\omega)}{1-\frac{1}{3} \Lambda(\omega)} ;
$$

$$
\begin{gathered}
\Lambda(\omega)=\frac{\omega_{p i}^{2}}{\omega_{d i}^{2}-\omega^{2}}+\frac{\omega_{p e-}^{2}}{\omega_{d e-}^{2}-\omega^{2}}+\frac{\omega_{p e+}^{2}}{\omega_{d e+}^{2}-\omega^{2}} ; \\
\mu=\frac{e N_{0}}{4 m} \frac{\varepsilon_{L}+2}{3} \frac{\Gamma(\omega)}{1-\frac{1}{3} \Lambda(\omega)} ; \\
\Gamma(\omega)=\frac{\alpha_{L}^{(+)}}{\omega_{d e+}^{2}-\omega^{2}}+\frac{\alpha_{L}^{(-)}}{\omega_{d e-}^{2}-\omega^{2}} .
\end{gathered}
$$

$\vec{\Pi}_{\omega}$ is Fourier-component of the quadratic dependence of the ponderomotive force (5) on the intensity of 
the electric field of a laser pulse. The value $\varepsilon(\omega)$ is the dielectric constant of a diatomic dielectric with an ion bond.

Maxwell's system of equations for Fouriercomponent of the electromagnetic field, taking into account the relation for the full polarization (8) can be represented as

$$
\begin{gathered}
\operatorname{rot} \vec{H}_{\omega}=-i k_{0} \varepsilon(\omega) \vec{E}_{\omega}+\frac{4 \pi}{c} \vec{j}_{\omega p o l}, \operatorname{rot} \vec{E}_{\omega}=i k_{0} \vec{H}_{\omega}, \\
\varepsilon(\omega) \operatorname{div} \vec{E}_{\omega}=4 \pi \rho_{\omega p o l}, \quad \operatorname{div} \vec{H}_{\omega}=0,
\end{gathered}
$$

$k_{0}=\omega / c$. The Fourier-components of the polarization currents and charges induced in the dielectric by the ponderomotive force of a laser pulse are described by the expressions

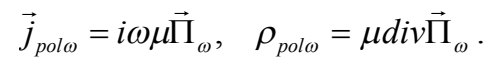

The resulting working system of equations makes it possible to investigate Cherenkov radiation in a wide variety of physical situations: the model of an infinite dielectric medium, dielectric waveguides and cavities.

\section{CHERENKOV RADIATION OF A LASER PULSE IN A DIELECTRIC WAVEGUIDE}

We consider the dielectric waveguide, made in the form of a homogeneous dielectric cylinder, the lateral surface of which is covered with a perfectly conductive metal film. A circularly polarized laser pulse with electric field components propagates along the axis of the waveguide

$$
\mathrm{E}_{0 \mathrm{x}}=\sqrt{\frac{\mathrm{I}_{0}}{2}} \psi(\mathrm{r}, \tau), \mathrm{E}_{0 \mathrm{y}}=\mathrm{iE}_{0 \mathrm{x}}, \psi=[\mathrm{R}(\mathrm{r}) \mathrm{T}(\tau)]^{1 / 2} .
$$

The function $\mathrm{R}(\mathrm{r})$ describes the radial profile of the laser pulse intensity $I_{0}=\left|\vec{E}_{0}\right|^{2}, R(0)=1, R(r=b)=0$, $b$ is the waveguide radius, the function $T(\tau)$ describes the longitudinal profile, $\tau=t-z / v_{g}, v_{g}$ is the group velocity, $\max T(\tau)=1, I_{0}$ is the maximum intensity.

From the system of Maxwell equations (10) the wave equation for the longitudinal Fourier component of the Cherenkov electric field follows

$$
\Delta \mathrm{E}_{\mathrm{z} \omega}+\mathrm{k}_{0}^{2} \varepsilon(\omega) \mathrm{E}_{\mathrm{z} \omega}=4 \pi\left(\frac{1}{\varepsilon(\omega)} \frac{\partial \rho_{\mathrm{pol} \omega}}{\partial \mathrm{z}}-\mathrm{i} \frac{\mathrm{k}_{0}}{\mathrm{c}} \mathrm{j}_{\mathrm{zpol} \omega}\right) .
$$

For a circularly polarized laser pulse (12), expressions for $\rho_{\text {pol }}, \vec{j}_{\text {polw }}$ take the form

$$
\begin{gathered}
\rho_{\text {pol } \omega}=\mu\left[\left(\Delta_{\perp}-\mathrm{k}_{\mathrm{g}}^{2}\right) \mathrm{I}_{\omega}(\mathrm{r})+\frac{\varepsilon_{\mathrm{L}}-1}{6} \Delta_{\perp} \mathrm{I}_{\omega}(\mathrm{r})\right] \mathrm{e}^{\mathrm{ikg} \mathrm{z}}, \\
\mathrm{j}_{z \text { pol } \omega}=-\omega \mathrm{k}_{\mathrm{g}} \mu \mathrm{I}_{\omega}(\mathrm{r}) \mathrm{e}^{\mathrm{ik}_{\mathrm{g}} \mathrm{z}},
\end{gathered}
$$

where $k_{g}=\omega / v_{g} \quad \Delta_{\perp}$ is the transverse part of Laplacian; $\mathrm{I}_{\omega}(r)$ is Fourier component of the intensity of the laser pulse field. The result of solving equation (13) is the following expression for the longitudinal component of the electric field in the form of a convolution

$$
E_{z}(r, \tau)=A_{0} \frac{1}{2 \pi} \int_{-\infty}^{\infty} T\left(\tau_{0}\right) G\left(r, \tau-\tau_{0}\right) d \tau_{0} .
$$

Here

$$
G\left(r, \tau-\tau_{0}\right)=\frac{1}{2 \pi} \int_{-\infty}^{\infty} G(r, \omega) e^{-i \omega\left(\tau-\tau_{0}\right)} d \omega
$$

is Green function. For further analysis, we will present the Green function in the form

$$
\begin{gathered}
G\left(r, \tau-\tau_{0}\right)=G_{l}\left(r, \tau-\tau_{0}\right)+G_{t r}\left(r, \tau-\tau_{0}\right), \\
G_{l}\left(r, \tau-\tau_{0}\right)=i \int_{-\infty}^{\infty} \omega \frac{\varepsilon(\omega)+2}{3} \frac{\Gamma(\omega)}{\varepsilon(\omega)} \Phi_{\omega}(r) e^{-i \omega\left(\tau-\tau_{0}\right)} d \omega, \\
G_{t r}\left(r, \tau-\tau_{0}\right)=-i \frac{\varepsilon_{L}-1}{6} \sum_{n=1}^{\infty} \delta_{n} J_{0}\left(\lambda_{n} \frac{r}{b}\right) S_{n}\left(\tau-\tau_{0}\right), \\
S_{n}\left(\tau-\tau_{0}\right)=i \int_{-\infty}^{\infty} \omega \frac{\varepsilon(\omega)+2}{3} \frac{k_{0}^{2} \Gamma(\omega)}{k_{n}^{2} \Delta_{n}(\omega)} e^{-i \omega\left(\tau-\tau_{0}\right)} d \omega,
\end{gathered}
$$

where

$$
\begin{gathered}
\delta_{n}=\frac{\lambda_{n}^{2}}{b^{2}} \frac{\rho_{n}}{N_{n}} ; N_{n}=\frac{b^{2}}{2} J_{1}^{2}\left(\lambda_{n}\right) ; \rho_{n}=\int_{0}^{b} R(r) J_{0}\left(\lambda_{n} \frac{r}{b}\right) r d r ; \\
\Delta_{\mathrm{n}}(\omega)=\mathrm{k}_{0}^{2} \varepsilon(\omega)-\mathrm{k}_{\mathrm{n}}^{2} ; \\
\Phi_{\omega}(r)=R(r)+\frac{\varepsilon_{L}-1}{6} \sum_{n=1}^{\infty} \frac{\delta_{n}}{k_{n}^{2}} J_{0}\left(\lambda_{n} \frac{r}{b}\right) ; k_{n}^{2}=k_{g}^{2}+\lambda_{n}^{2} / b^{2} .
\end{gathered}
$$

The Green function actually describes the structure of the wakefield in a dielectric medium excited by a laser pulse with a $\delta$-shaped longitudinal intensity profile. Moreover, the term $G_{l}\left(r, \tau-\tau_{0}\right)$ takes into account the excitation of potential longitudinal oscillations of the ion dielectric, and the term $G_{t r}\left(r, \tau-\tau_{0}\right)$ describes the excitation of transverse electromagnetic waves.

\subsection{DISPERSION PROPERTIES OF ION DIELECTRIC WAVEGUIDE}

Let us now briefly discuss the question of the propagation of electromagnetic waves in an ion dielectric waveguide. Dispersion equations for potential longitudinal oscillations and electromagnetic waves have the form

$$
\begin{gathered}
\varepsilon(\omega)=0, \\
\frac{\omega^{2}}{c^{2}} \varepsilon(\omega)-k_{z}^{2}-\frac{\lambda_{n}^{2}}{b^{2}}=0,
\end{gathered}
$$

$k_{z}$ is longitudinal wave number. The dielectric constant is described by the formula (9). The expression for the dielectric constant can be represented as [5]

$$
\varepsilon(\omega)=\frac{\left(\omega^{2}-\omega_{L i}^{2}\right)\left(\omega^{2}-\omega_{L e_{-}}^{2}\right)\left(\omega^{2}-\omega_{L e+}^{2}\right)}{\left(\omega^{2}-\omega_{T i}^{2}\right)\left(\omega^{2}-\omega_{T e-}^{2}\right)\left(\omega^{2}-\omega_{T e+}^{2}\right)} .
$$

The frequency $\omega_{L i}$ is the frequency of longitudinal optical phonons and belongs to the infrared frequency range. Frequencies $\omega_{L e( \pm)}$ are the frequencies of longitudinal polarization electron oscillations and are in the optical or even ultraviolet frequency ranges. The frequencies $\omega_{T i}, \omega_{T e( \pm)}$ are the absorption lines of the electromagnetic waves of an ion crystal. In the vicinity of these frequencies, the imaginary part of the dielectric constant and, accordingly, the energy losses of electromagnetic waves increase greatly. The frequency of absorption by the ion subsystem $\omega_{T i}$ is the frequency of transverse optical phonons.

In total, there are three branches of longitudinal oscillations $\omega=\omega_{L i}, \omega_{L e( \pm)}$ and four branches of electro- 
magnetic waves [5]. The low-frequency branch $\omega=\omega_{L i}$ corresponds to the longitudinal optical phonons, and the other two branches $\omega=\omega_{L e( \pm)}$ are frequencies of the polarization electron oscillations. As for the electromagnetic branches, the lowest frequency (ion) branch1 is in the infrared and microwave ranges $\omega_{T i}>\omega_{1}\left(k_{z}\right)>\omega_{c i-}, \quad \omega_{c i-}=\lambda_{n} c / b \sqrt{\varepsilon_{s t}}$ is low ion cutoff frequency

$$
\varepsilon_{s t}=\frac{\omega_{L i}^{2}}{\omega_{T i}^{2}} \varepsilon_{o p t}, \varepsilon_{o p t}=\frac{\omega_{L e-}^{2}}{\omega_{T e-}^{2}} \frac{\omega_{L e+}^{2}}{\omega_{T}^{2}},
$$

$\varepsilon_{s t}$ is the static dielectric constant. In the frequency range $\omega_{T i}>>\omega>\omega_{c i-}$, the dispersion curve has a linear plot $\omega=k_{z} c / \sqrt{\varepsilon_{s t}}$. The frequencies of the electromagnetic branch 2 are within $\omega_{T e_{-}}>\omega_{2}\left(k_{z}\right)>\omega_{c i+} \approx \omega_{L i}$, $\omega_{c i+}$ is high frequency ion cutoff frequency The lowfrequency section of this branch corresponds to the infrared frequency range and the high-frequency region corresponds to the optical one. This branch also has a linear dispersion region $\omega=k_{z} c / \sqrt{\varepsilon_{\text {opt }}}, \varepsilon_{\text {opt }}$ is the optic dielectric constant. The tilt angle of this line exceeds the tilt angle of the straight section of branch 1. And finally, branches 3 and 4 are purely electron branches and locate in the optical and ultraviolet frequency ranges. The phase velocity of electromagnetic waves belonging to the fourth branch exceeds the speed of light and in the limiting case approaches it.

\subsection{CALCULATION OF GREEN'S FUNCTION}

The Green function (18) contains two terms that describe the excitation of longitudinal potential oscillations and electromagnetic waves. The potential Green's function $G_{l}\left(r, \tau-\tau_{0}\right)$ has only simple poles, which are the zeros of the dielectric constant $\varepsilon(\omega)=0$. The frequency spectrum of longitudinal oscillations contains the frequency of longitudinal optical phonons $\omega_{L i}$ and the frequencies $\omega_{L e(\mp)}$ of electron polarization oscillations. Below we restrict ourselves to the study of wake fields in the infrared and lower frequency ranges.

Calculating the residues in the integral $G_{l}\left(r, \tau-\tau_{0}\right)$ at the poles $\omega= \pm \omega_{L i}-i 0$, we find the following for the potential Green function expression

$$
G_{l}=\frac{4 \pi}{3} \omega_{L i}^{2} \frac{1}{\varepsilon_{e f f}} \Gamma_{s t} \Phi_{i}(r) \vartheta\left(\tau-\tau_{0}\right) \cos \omega_{L i}\left(\tau-\tau_{0}\right),
$$

where $\vartheta\left(\tau-\tau_{0}\right)$ is the Heaviside function;

$$
\begin{gathered}
\varepsilon_{e f f}=\frac{\varepsilon_{s t} \varepsilon_{o p t}}{\varepsilon_{s t}-\varepsilon_{o p t}} ; k_{i}=\omega_{L i} / v_{g} ; \Gamma_{s t}=\frac{\alpha_{L}^{(+)}}{\omega_{d e+}^{2}}+\frac{\alpha_{L}^{(-)}}{\omega_{d e-}^{2}} ; \\
\Phi_{i}(r)=R(r)+\frac{\varepsilon_{L}-1}{6} k_{i}^{2} \int_{0}^{b} G_{r}\left(r, r_{0}\right) R\left(r_{0}\right) r_{0} d r_{0} ; \\
G_{r}\left(r, r_{0}\right)=\frac{1}{I_{0}\left(k_{i} b\right)}\left\{\begin{array}{l}
I_{0}\left(k_{i} r\right) \Delta_{0}\left(k_{i} r_{0}\right), r<r_{0}, \\
I_{0}\left(k_{i} r_{0}\right) \Delta_{0}\left(k_{i} r\right), \quad r>r_{0} ;
\end{array}\right. \\
\Delta_{0}\left(k_{i} r\right)=I_{0}\left(k_{i} r\right) K_{0}\left(k_{i} b\right)-I_{0}\left(k_{i} b\right) K_{0}\left(k_{i} r\right) .
\end{gathered}
$$

Term in the total Green's function $G_{t r}\left(r, \tau-\tau_{0}\right)$ describes the Cherenkov excitation of the eigen electromagnetic waves of the dielectric waveguide. Integrands of Fourier integrals $S_{n}\left(\tau-\tau_{0}\right)$ contain only simple poles, which are the roots of the equation

$$
\Delta_{n}(\omega)=0 \text {. }
$$

As we are interested in the infrared (microwave) frequency range equation three pairs of roots. Two of them are located on the real axis

$$
\begin{gathered}
\omega= \pm \omega_{i n}-i 0, \quad \omega_{i n}=\frac{\lambda_{n} v_{g}}{b} \frac{1}{\sqrt{\beta_{g}^{2} \varepsilon_{s t}-1}}, \\
\omega= \pm \omega_{e n}-i 0, \omega_{e n}=\sqrt{\omega_{T e+} \omega_{T e-}}\left(\frac{\omega_{n}^{2}}{\varepsilon_{o p t} \omega_{T e}^{2}-\omega_{L e}^{2}}\right)^{1 / 4}, \\
\omega_{T e}^{2}=\omega_{T e-}^{2}+\omega_{T e+}^{2}, \omega_{L e}^{2}=\omega_{L e-}^{2}+\omega_{L e+}^{2}, \omega_{n}=\lambda_{n} c / b,
\end{gathered}
$$

and one pair $\omega= \pm i \omega_{e n}$ on the imaginary axis. Calculating the residues in these poles we find the expression for the Green function

$$
\begin{aligned}
& G_{t r}=-\frac{\pi}{9} e_{s t} \Gamma_{s t} \vartheta\left(\tau-\tau_{0}\right) \sum_{n=1}^{\infty} \omega_{i n}^{2} \sigma_{n} J_{0}\left(\lambda_{n} \frac{r}{b}\right) \cos \omega_{i n}\left(\tau-\tau_{0}\right)- \\
& -\frac{\pi}{18} e_{o p t} \Gamma_{s t} \sum_{n=1}^{\infty} \omega_{e n}^{2} \sigma_{n} J_{0}\left(\lambda_{n} \frac{r}{b}\right)\left[\vartheta\left(\tau-\tau_{0}\right) \cos \omega_{e n}\left(\tau-\tau_{0}\right)-\right. \\
& \left.-\frac{1}{2} \operatorname{sign}\left(\tau-\tau_{0}\right) \exp \left(-\omega_{e n}\left|\tau-\tau_{0}\right|\right)\right],
\end{aligned}
$$

where

$\sigma_{n}=\frac{\rho_{n}}{N_{n}} ; e_{s t}=\frac{\left(\varepsilon_{L}-1\right)\left(\varepsilon_{s t}+2\right)}{\varepsilon_{s t}} ; e_{o p t}=\frac{\left(\varepsilon_{L}-1\right)\left(\varepsilon_{o p t}+2\right)}{\varepsilon_{o p t}}$.

The first term in the expression for the electromagnetic Green's function (26) describes the electric field in the microwave (terahertz) frequency range $\omega_{L i}^{2} \gg \omega_{i n}^{2}$ (ion branch 1) and is a set of eigen electromagnetic waves with frequencies $\omega_{i n}$. The second term in expression (26) describes a purely electron electromagnetic field and belongs to branch 2 in the infrared frequency range $\omega_{T e-}^{2}>>\omega_{e n}^{2} \gg>\omega_{L i}^{2}$. The longitudinal structure of this field is more complicated. Each radial harmonic contains a wake monochromatic wave, as well as a bipolar antisymmetric solitary pulse. Moreover, the height of this pulse is exactly two times smaller than the amplitude of the wake wave. Since the amplitudes of the waves entering the Green function are proportional to the square of their frequencies, the electron electromagnetic waves will have a larger amplitude compared to the ion waves.

\subsection{THE EXCITATION OF WAKE FIELD BY LASER PULSE}

The wakefield excited by a laser pulse is described by convolution (16), in which the Green function is the key element. We first consider the excitation of longitudinal optical phonons. Using the potential polarization part of the Green function, we obtain the following expression for the wake field of longitudinal optical phonons

$$
E_{i z}(r, \tau)=E_{L i} \Phi_{i}(r) Z\left(\omega_{L i} \tau\right)
$$


where $Z(\omega \tau)=\frac{1}{t_{L}} \int_{-\infty}^{\tau} T\left(\tau_{0} . / t_{L}.\right) \cos \omega\left(\tau-\tau_{0}\right) d \tau_{0}$

$$
E_{L i}=\frac{2 \pi}{9} \frac{\left(\varepsilon_{\text {opt }}+2\right)}{\varepsilon_{\text {eff }}} \frac{e \omega_{L i}^{2}}{v_{g}^{2}} \frac{v_{g} t_{l}}{r_{c l}} \kappa_{L} a_{0}^{2}
$$

$t_{L}$ is characteristic duration of a laser pulse.

$$
\kappa_{L}=\Gamma_{s t} N_{0} \omega_{L}^{2}, r_{c l}=e^{2} / m c^{2}, a_{0}^{2}=\left(\frac{e}{m c \omega_{L}}\right)^{2} I_{0} .
$$

The wake function $Z(\omega \tau)$ describes the distribution of the wakefield on frequency $\omega$ in the longitudinal direction at each moment of time. We will consider a laser pulse with a symmetric longitudinal profile $T\left(\tau_{0}\right)=T\left(-\tau_{0}\right)$. The wake function is conveniently represented as

$$
Z(\omega \tau)=\widehat{T}(\Omega) \vartheta(\tau) \cos \omega \tau-X(\bar{\tau}),
$$

where $\Omega=\omega t_{L} ; \bar{\tau}=\tau / t_{L} ; \widehat{T}(\Omega)=2 \int_{0}^{\infty} T(s) \cos (\Omega s) d s$;

$$
X(\bar{\tau})=\operatorname{sign} \tau \int_{|\bar{\tau}|}^{\infty} T(s) \cos \Omega(|\bar{\tau}|-s) d s .
$$

The first term in (28) describes the wake wave propagating behind the laser pulse. The second term in (28) describes a bipolar antisymmetric pulse of a polarization field localized in the region of a laser pulse. The field of this pulse decreases and tends to zero with increasing distance from the laser pulse.

Behind a laser pulse, the wakefield of longitudinal optical phonons has the form of a monochromatic wave

$$
E_{i z}(r, \tau)=E_{L i} \Phi_{i}(r) \hat{T}\left(\Omega_{L i}\right) \cos \omega_{L i} \tau, \quad \Omega_{L i}=\omega_{L i} t_{L} .
$$

Let us give expressions for the Fourier amplitude $\widehat{T}(\Omega)$ for Gaussian model longitudinal profiles of a laser pulse

$$
T\left(\tau_{0} / t_{L}\right)=\exp \left(-\tau_{0}^{2} / t_{L}^{2}\right), f(\Omega)=\sqrt{\pi} \exp \left(-\Omega^{2} / 4\right) .
$$

Longitudinal optical phonons are most efficiently radiated when the coherence condition $\omega_{L i} t_{L} \leq 1$ is satisfied. If the condition $\omega_{L i} t_{L}>>1$ is satisfied, then the longitudinal optical phonons are radiated incoherently and the amplitude of the wake wave is exponentially small. We present the expressions for the amplitudes of longitudinal optical phonons $E_{L i}$. For two alkaline halide ion dielectrics: sodium chloride $\mathrm{NaCl}$ and potassium iodide $\mathrm{KI}$, we have

$$
E_{L i}(\mathrm{NaCl})=7.4 \cdot 10^{4} \frac{N_{L}}{\lambda_{L}(\mu \mathrm{m})} a_{0}^{2}(\mathrm{~V} / \mathrm{cm}),
$$

where $N_{L}$ is number of wavelengths in the laser pulse; $f_{L i}=7.62 \cdot 10^{12} \mathrm{~Hz}$ is frequency of longitudinal optical phonons. In the case of potassium iodide, we obtain

$$
E_{L i}(K I)=1.16 \cdot 10^{4} \frac{N_{L}}{\lambda_{L}(\mu m)} a_{0}^{2}(\mathrm{~V} / \mathrm{cm}),
$$

$f_{T i}=4 \cdot 10^{12} \mathrm{~Hz}$. The amplitude of the longitudinal optical phonons and their frequency are lower than in the case of potassium chloride. For $\lambda_{L}=1 \mu \mathrm{m}, N=30$ and $a_{0}=1 \quad$ we find $E_{L i}(\mathrm{NaCl})=2.2 \mathrm{MV} / \mathrm{cm}$, $E_{L i}(K I)=0.35 \mathrm{MV} / \mathrm{cm}$.
Let us now consider the excitation of electromagnetic waves by a laser pulse. Taking advantage of the electromagnetic Green's function, we obtain the wake electromagnetic field as a superposition of radial harmonics

$$
\begin{gathered}
E_{t z}(r, \tau)=-E_{t i} \sum_{n=1}^{\infty} \frac{\omega_{i n}^{2}}{\omega_{0}^{2}} \sigma_{n} J_{0}\left(\lambda_{n} \frac{r}{b}\right) Z\left(\omega_{i n} \tau\right)- \\
-E_{t e} \sum_{n=1}^{\infty} \frac{\omega_{e n}^{2}}{\omega_{0}^{2}} \sigma_{n} J_{0}\left(\lambda_{n} \frac{r}{b}\right)\left[Z\left(\omega_{e n} \tau\right)-\frac{1}{2} Y\left(\omega_{e n} \tau\right)\right],
\end{gathered}
$$

where

$$
\begin{gathered}
Y\left(\omega_{e n} \tau\right)=\int_{-\infty}^{\infty} T\left(\tau_{0} / t_{L}\right) \operatorname{sign}\left(\tau-\tau_{0}\right) e^{-\omega_{e n}\left|\tau-\tau_{0}\right|} d \tau_{0} ; \\
E_{t i}=\frac{\pi}{18} e_{s t} \frac{\varepsilon_{o p t}+2}{3} \kappa_{L} \frac{v_{g} t_{L}}{r_{c l}} \frac{e \omega_{0}^{2}}{v_{g}^{2}} a_{0}^{2}, \quad \omega_{0}=c / b ; \\
E_{t i}=\frac{\pi}{36} e_{o p t} \frac{\varepsilon_{o p t}+2}{3} \kappa_{L} \frac{v_{g} t_{L}}{r_{c l}} \frac{e \omega_{0}^{2}}{v_{g}^{2}} a_{0}^{2} .
\end{gathered}
$$

Behind the laser pulse $\tau / t_{L} \gg 1, \omega_{n i, e} \tau \gg 1$, the pulse fields are negligible and only the set of eigen waves of the dielectric waveguide remains

$$
\begin{array}{r}
E_{t z}(r, \tau)=-\sqrt{\pi} \sum_{n=1}^{\infty} \sigma_{n} J_{0}\left(\lambda_{n} \frac{r}{b}\right)\left[E_{t i} \frac{\omega_{i n}^{2}}{\omega_{0}^{2}} e^{-\frac{\omega_{i n}^{2} t_{L}^{2}}{4}} \cos \left(\omega_{i n} \tau\right)+\right. \\
\left.+E_{t e} \frac{\omega_{e n}^{2}}{\omega_{0}^{2}} e^{-\frac{\omega_{e n}^{2} t_{L}^{2}}{4}} \cos \left(\omega_{e n} \tau\right)\right] .
\end{array}
$$

Let us consider, for example, a laser pulse that has a Gaussian profile both in the longitudinal direction and in the transverse one

$$
R\left(r_{0} / r_{L}\right)=\exp \left(-r_{0}^{2} / r_{L}^{2}\right)
$$

moreover, the radius of the laser pulse is small compared with the radius of the dielectric waveguide $r_{L}<<b$. In this case, for the expansion coefficients in the series (29) we have

$$
\sigma_{n}=\frac{r_{L}^{2}}{b^{2}} \frac{1}{J_{1}^{2}\left(\lambda_{n}\right)} \exp \left(-\frac{\lambda_{n}^{2} r_{L}^{2}}{4 b^{2}}\right) .
$$

Accordingly, for the wake electromagnetic field instead of (29) we obtain

$$
\begin{gathered}
E_{t z}(r, \tau)=-\sqrt{\pi} \sum_{n=1}^{\infty} \sigma_{n} J_{0}\left(\lambda_{n} \frac{r}{b}\right)\left[E_{t i} \frac{\omega_{i n}^{2}}{\omega_{0}^{2}} e^{-\frac{\omega_{i n}^{2} \tau_{L}^{2}}{4}} \cos \left(\omega_{i n} \tau\right)+\right. \\
\left.+E_{t e} \frac{\omega_{e n}^{2}}{\omega_{0}^{2}} e^{-\frac{\omega_{e n}^{2} t_{L}^{2}}{4}} \cos \left(\omega_{e n} \tau\right)\right] .
\end{gathered}
$$

Amplitudes of wake electromagnetic waves are proportional to the square of their frequencies. Therefore, a short laser pulse will predominantly excite electron electromagnetic waves, since their frequencies greatly exceed the frequencies of ion electromagnetic waves $\omega_{e n}>>\omega_{i n}$. But the number of these waves is limited by inequality $\omega_{e n} t_{L} \leq 1$. If the laser pulse is long at the scale of the minimum period of electron electromagnetic waves $\omega_{e 1} t_{L}>1$, but short compared with the periods of ion electromagnetic waves $\omega_{i n} t_{L}<<1$, then lowfrequency ion electromagnetic waves will be most ef- 
fectively excited. Under these conditions, only lowfrequency waves are emitted coherently by a laser pulse.

\section{CONCLUSIONS}

In this work, the process of excitation of wake Cerenkov radiation by a laser pulse in an ion dielectric waveguide is investigated. For definiteness, a diatomic ion crystal medium is considered. The nonlinear electric polarization of the ion dielectric medium, induced by the ponderomotive force with the side of the laser pulse, is determined. The total electric polarization in the ion dielectric includes the electron polarization of the electron shells of ions of opposite charges, as well as the ion polarization proper, due to the displacement of ions in the electric field. A system of three strongly coupled linear oscillator equations is obtained, which describes the excitation of partial electric polarizations of an ion dielectric by a ponderomotive force from the side of a laser pulse. The solution of these equations is obtained and the complete polarization in a diatomic ion dielectric medium is determined. Accordingly, expressions are obtained for polarization charges and currents, which, in turn, are the source of Cerenkov wake waves. The frequency spectrum and the space-time structure of the Cherenkov wake field, excited by a laser pulse in an ion dielectric waveguide, are determined. It is shown that in the infrared (microwave) frequency range, the excited wake electric field consists of a potential field of longitudinal optical phonons and a set of eigen wake electromagnetic waves of a dielectric waveguide. The dielectric constant in the infrared (microwave) frequency range in ion dielectrics always exceeds the dielectric constant in the optical range. Therefore, the condition of the Cherenkov radiation of a laser pulse in ion dielectrics is always satisfied.

\section{REFERENCES}

1. S.A. Akhmanov, V.A. Fold. Optics of femtosecond laser pulses. M.: "Nauka", 1988, 388 p.

2. V.L. Ginzburg, V.I. Tsytovich. Transition radiation and transition scattering. M.: "Nauka", 1964, p. 360 .

3. V.A. Balakirev, I.N. Onishchenko. Cherenkov radiation of a laser pulse in a dielectric waveguide // Problems of Atomic Science and Technology. Series “Plasma Physics”. 2018, № 6, p. 147-151.

4. V.A. Balakirev, I.N. Onishchenko. Wakefield excitation by a laser pulse in a dielectric medium // Problems of Atomic Science and Technology. Series "Plasma Electronics and New Methods of Acceleration”. 2018, № 4, p. 76-82.

5. V.A. Balakirev, I.N. Onishchenko. Cherenkov radiation of a laser pulse in ion dielectrics // Problems of Atomic Science and Technology. Series "Plasma Electronics and New Methods of Acceleration". 2019, № 4, p. 39-47.

6. T. Tajima, J.M. Dawson. Laser electron acceleration // Phys. Rev. Letter. 1979, v. 43, № 4, p. 267-270.

7. M. Born, H. Kun. Dynamic theory of cristal lattice. M.: "Foreign literature", 1958, 488 p.

8. J. Sleter. Dielectrics, semiconductors, metals. M.: "Mir", 1969, 647 p.

9. N. Ashcroft, N. Mermin. Solid State Physics. M.: "Mir", 1978, v. 2, 392 p.

10. V.A. Balakirev, G.V. Sydel'nikov. Physical mechanisms for transition radiation of electromagnetic pulses // Technical Physics. 1999, v. 44, № 10, p. 1209-1214.

Article received 22.10.2019

\section{ВОЗБУЖДЕНИЕ КИЛЬВАТЕРНЫХ ВОЛН ЛАЗЕРНЫМ ИМПУЛЬСОМ В ИОННОМ ДИЭЛЕКТРИКЕ}

\section{В.А. Балакирев, И.Н. Онищенко}

Исследован процесс возбуждения черенковского электромагнитного излучения лазерным импульсом в ионном диэлектрическом волноводе. Показано, что возбуждаемое поле состоит из потенциального поляризационного электрического поля продольных оптических фононов и набора собственных электромагнитных волн ионного диэлектрического волновода.

\section{ЗБУДЖЕННЯ КІЛЬВАТЕРНИХ ХВИЛЬ ЛАЗЕРНИМ ІМПУЛЬСОМ В ІОННОМУ ДІЕЛЕКТРИКУ В.О. Балакірєв, І.М. Оніщенко}

Досліджено процес збудження черенковського електромагнітного поля лазерним імпульсом в іонному діелектричному хвилеводі. Показано, що збуджуване поле складається з потенціального поляризаційного електричного поля поздовжніх оптичних фононів та набору власних електромагнітних хвиль іонного діелектричного хвилеводу. 